\title{
Competitividad turística de los municipios de Málaga, Capitanejo, Concepción y San Andrés, de la provincia García Rovira
}

Tourism competitiveness in the municipalities of Málaga, Capitanejo, Concepción and San Andrés, in the García Rovira Province

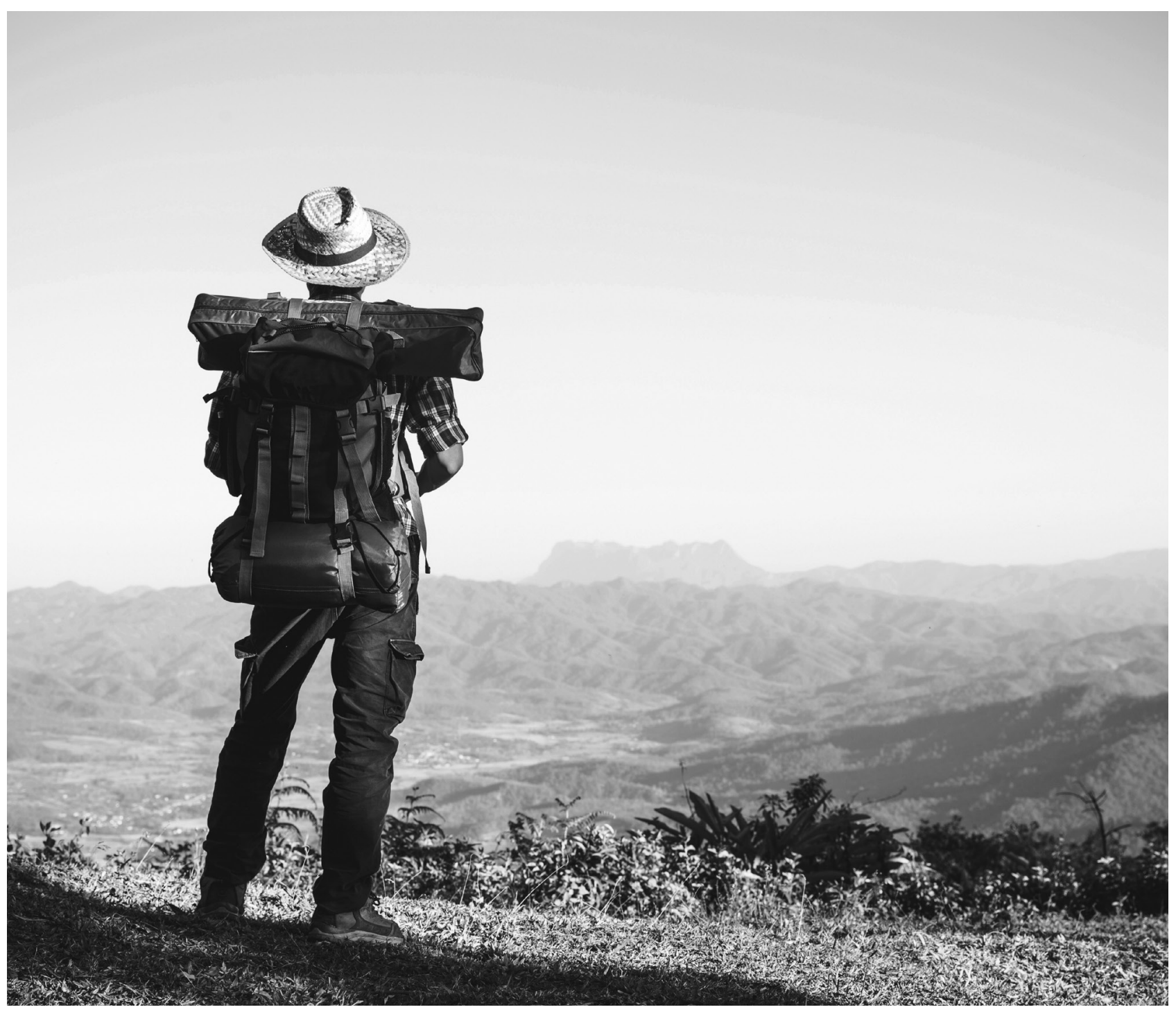




\title{
Competitividad turística de los municipios de Málaga, Capitanejo, Concepción y San Andrés, de la provincia García Rovira ${ }^{1}$
}

\section{Tourism competitiveness in the municipalities of Málaga, Capitanejo, Concepción and San Andrés, in the García Rovira Province}

\author{
Wendy Carolina Jiménez Roa ${ }^{2}$, Paola Andrea Villamizar Pinto ${ }^{3}$, \\ Oscar Julián Sanabria Ospino ${ }^{4}$
}

Artículo recibido en febrero 20 de 2019; artículo aceptado en abril 5 de 2019.

\begin{abstract}
Este artículo puede compartirse bajo la Licencia Creative Commons Atribución-NoComercial-Compartir Igual 4.0 Internacional
y se referencia usando el siguiente formato: Jiménez, W. C., Villamizar, P. A. \& Sanabria, O. J. (2019). Competitividad turística de los municipios de Málaga, Capitanejo, Concepción y San Andrés, de la provincia García Rovira. I+D Revista de Investigaciones,
\end{abstract}

14 (2), 64-74. DOI: https://doi.org/10.33304/revinv.v14n2-2019006

\begin{abstract}
Resumen
En este documento se analiza la competitividad del sector turismo en los municipios de Málaga, Capitanejo, Concepción y San Andrés, de la provincia García Rovira, del departamento de Santander. Se identificaron sus atractivos turísticos, potencial y estado de infraestructura actual. Se realizó trabajo de campo, para recolectar información acerca del turismo en la región de estudio, y se utilizó para ello una encuesta aplicada a propietarios de establecimientos turísticos. A partir de los datos obtenidos, se realizó un diagnóstico basado en la matriz DOFA, en el que se presentan de manera desglosada las fortalezas, las debilidades, las oportunidades y las amenazas que posee la región. Esto se hizo enfrentando factores internos y externos, con la intención de contrarrestar los factores poco favorables con aspectos positivos. Los resultados indican que la competitividad del sector turístico en esta región es baja. Aunque tiene alto potencial, debido a la variedad de atractivos turísticos naturales que posee, no usa eficientemente estos recursos y no obtiene su rentabilidad.
\end{abstract}

Palabras clave: Turismo, competitividad, ecoturismo, infraestructura, Santander.

\footnotetext{
Abstract

The document analyzes the competitiveness of the tourism sector in the municipalities of Málaga, Capitanejo, Concepción and San Andrés, in the García Rovira province of the department of Santander. Their tourism attractions,

1. Artículo de investigación exploratoria descriptiva, con enfoque cualitativo, resultado de un proyecto de investigación culminado, perteneciente al área de negocios y dirección empresarial, subárea del turismo, desarrollado en la Universidad de Investigación y Desarrollo de la ciudad de Bucaramanga (Colombia). Dirección: calle 9 n. ${ }^{\circ} 23-55$, PBX: 6352525. Fecha de inicio: febrero de 2017; fecha de terminación: noviembre de 2017.

2. Profesional en Negocios Internacionales. Universidad de Investigación y Desarrollo (UDI), Bucaramanga (Colombia). Dirección: calle 9 n. ${ }^{\circ} 23-55$, PBX: 6352525. ORCID ID: https://orcid.org/0000-0002-6331-1506. Correo electrónico institucional: wjimenez1@udi.edu.co.

3. Profesional en Negocios Internacionales. Universidad de Investigación y Desarrollo (UDI), Bucaramanga (Colombia). Dirección: calle 9 n. ${ }^{\circ} 23-55$, PBX: 6352525. ORCID ID: https://orcid.org/0000-0002-3491-5343. Correo electrónico: andreita11-12@hotmail.com.

4. Magíster en Derecho, de la Universidad Santo Tomás. Notario único, abogado especialista en Derecho Comercial y Derecho Tributario. Notario de la Notaría Única de Zapatoca (Colombia). Dirección: carrera 10 n. 19-30, PBX: 6252247. ORCID ID: https://orcid.org/0000-0002-7679-130X. Correo electrónico: oscarsan35@gmail.com.
} 
their potential, and their current infrastructure status were identified. Fieldwork was carried out to collect information about tourism in the regions studied and a survey was applied to owners of tourist establishments. Based on the collected data, a diagnosis was made based on the DOFA Matrix, where the strengths, weaknesses, opportunities and threats of the region are presented in a disaggregated manner, confronting internal and external factors in order to counterbalance the unfavorable factors with positives ones. The results indicate that the competitiveness of the tourism sector in this region is low. Even though the region has high potential, due to the variety of natural tourist attractions, it does not use these resources efficiently and does not obtain profits.

Keywords: Tourism; competitiveness, ecotourism, infrastructure, Santander.

\section{Introducción}

En épocas contemporáneas, el turismo se ha posicionado como uno de los sectores más influyentes en la vida del hombre. Por esto, su incentivo es prioridad para los países, y Colombia no debe ser la excepción. Por medio del turismo, se fomentan la recreación, la competitividad y el crecimiento económico de un país, región, provincia, departamento o municipio (Hong, 2009).

Colombia es un país destacado por el turismo de tipo litoral (sol y playa); sin embargo, hasta hace pocos años ha identificado su potencial en otros tipos de turismo, como el turismo de montaña o el ecoturismo (Botero \& Zielinski, 2010). Para tal fin, el departamento de Santander cuenta con grandes atractivos turísticos relacionados con su infraestructura coloquial, como los que se encuentran en el municipio de Barichara, en la provincia de Guanentá. Dentro de estos atractivos se resaltan el Bioparque Móncara, el Camino Real, la calle del Mirador, la iglesia de estilo romántico, la Capilla de Jesús y el parque natural de la Chorrera. En el departamento se han dejado en el olvido provincias como la de García Rovira, región en la que se centra esta investigación.

Por tanto, en el presente documento se intenta analizar la competitividad del sector turismo en los principales municipios de la provincia García Rovira, con el fin de tener una visión del turismo en esta región. Esto resulta de utilidad para el desarrollo de programas de financiamiento en áreas puntuales. Programas que pueden ser realizados por los entes gubernamentales o por instituciones privadas. Para lograr esto, se identifican las condiciones actuales del sector en los municipios de Málaga, Capitanejo, Concepción y San Andrés. Se reconoce el potencial turístico de la región y finalmente se observan los niveles de competitividad.

Para la recolección de información, se usan fuentes primarias y secundarias. Las primeras se llevaron a cabo con trabajo de campo, a través de visitas y encuestas realizadas a una muestra específica de la población de estudio, así como mediante consulta a expertos.
Las segundas se refieren a páginas gubernamentales oficiales de los distintos municipios. Con la información recolectada, se realiza una matriz DOFA con los factores que afectan a la provincia. Se comparan y enfrentan las variantes positivas con las negativas, y asimismo se contrastan las debilidades y amenazas con las oportunidades y fortalezas. De esta forma, se intenta determinar si la región es viable para el turismo y merece estudios de mayor profundidad en el futuro, o si, por el contrario, no está cumpliendo con las expectativas para ser considerada como un destino atractivo para el turismo.

El documento inicia con una revisión de la literatura sobre el turismo y la competitividad. Posteriormente se presenta la metodología utilizada, seguida de los resultados. Y finalmente se exponen las conclusiones.

\section{Evolución del sector turismo}

El inicio del turismo en el ámbito mundial data del año 1841, cuando, en Inglaterra, un grupo de personas de la "Sociedad de la Esperanza" viajaba en tren desde Leicester con destino a Loughborogh para conocer nuevos pueblos y personas (Cardoso Jiménez, 2006). A principios del presente siglo, con el descubrimiento del automóvil, el avión y la navegación, se incrementa notoriamente el turismo. Sin embargo, las guerras en Europa y la crisis económica mundial paralizan su auge en esa época (Faraldo \& Rodríguez-López, 2013). Hacia el año 1995, con la Organización Mundial del Turismo (OMT), se ve reflejado un aumento notorio en el turismo, ya que para ese entonces 125 países eran miembros. La OMT contaba, además, con 250 miembros afiliados (Organización Mundial del Turismo, 2015).

Para finales de la década de los setenta, Colombia era el país suramericano con mayor crecimiento turístico. Logró tasas mayores al $17 \%$ anual por más de un decenio. Pero es en 1931, mediante la Ley 86 , cuando se da inicio al turismo en el país (Zuñiga Collazos, 2015). Según estadísticas de la Organización Mundial de Comercio (2016), para el año 2014 Europa ocupaba el primer lugar en el ámbito 
mundial en visitas turísticas; América, el tercer puesto, y Colombia, el quinto en América, con una participación de $0,23 \%$ del total de llegadas en el mundo.

En Colombia, crece vertiginosamente el turismo, y se proyecta como uno de los renglones fuertes para la economía. Esto, dado a que este país ocupa el segundo puesto en el ámbito mundial en biodiversidad, cuenta con dos océanos y tiene más de 10.000 sitios turísticos para visitar (Porras Celis \& Ruiz Pico, 2005).

Por su parte, Santander se considera como una de las regiones del futuro de Suramérica, según Financial Times (2016). Esta organización midió el PIB, el desempleo, los macroproyectos y el crecimiento anual. En sus resultados, destacó el buen momento económico y las oportunidades de inversión que, por tradición, ha presentado Santander.

De igual modo, la provincia García Rovira se destaca por su gastronomía. Destacan allí la actividad dulcera, las colaciones, los quesos y las tradicionales panuchas. Los municipios de esta provincia son Macaravita, Málaga, Molagavita, San Andrés, San José de Miranda, San Miguel, Guaca, Capitanejo, Carcasí, Enciso, Concepción y Cerrito.

Cabe resaltar que las tendencias en el turismo en los últimos años se han unificado rápidamente. Propuestas de turismo emergente, como turismo rural, turismo verde, ecoturismo, turismo ecológico y sostenible son el resultado de la cultura y el proceso de concienciación sobre la explotación de los recursos naturales. Estas prácticas tienen sus cimientos en Europa, y se están convirtiendo en modelos de negocio sustentable en diferentes partes alrededor del mundo (García, Ramírez \& González, 2018).

\section{Competitividad}

La competitividad se define como la habilidad de un país para desarrollar y sostener en el largo plazo un valor económico añadido, en relación con sus competidores (Fayos-Solá, 1994). En este sentido, al enfocar el concepto en un sector, se puede decir que la competitividad es la capacidad de un sector o de una industria para alcanzar sus objetivos de forma superior y sostenible, con respecto a los demás sectores en los ámbitos local, nacional e internacional (González \& Mendieta, 2009; Rice, 2018).

En este sentido, teniendo en cuenta que el sector turismo compite con otros sectores, se hace necesario que la política turística de los países evolucione y se centre en el logro de competitividad, tanto para las empresas como para las regiones turísticas que deben adaptarse a las necesidades del entorno actual. Ello para que las inversiones privadas y públicas alcancen las rentabilidades turísticas y mejoren la competitividad (Fayos-Solá, 1994). Por ello, es importante que Colombia no sea ajena al tema y tienda, en cambio, a incentivar el sector turismo en las diferentes regiones del país.

La implementación de estrategias de competitividad para el desarrollo económico de las regiones de Colombia, y en el caso específico de este estudio, en la región de Santander, ha sido de interés general (Castro \& Robles, 2018; Manrique, 2013). Esto porque, según el Índice de Competitividad Global del Foro Económico Mundial, Colombia ocupa el puesto 60 entre 140 países (World Economic Forum, 2018), lo que sugiere la necesidad de introducir una política de competitividad nacional sobre la que se deben diseñar las estrategias para el desarrollo en empresas, municipios y otros entes gubernamentales.

\section{Indicadores de competitividad del sector}

En el departamento de Santander se ha empezado a tener un enfoque más turístico en las diferentes regiones. Ejemplo de ello es el trabajo que está realizando la Agencia de Desarrollo Económico Local (ADEL) con la provincia García Rovira, con miras a promocionar más el territorio. Gracias a esto, y a los incentivos que se han otorgado poco a poco en los últimos años, Santander, en el año 2016, se posicionó como la cuarta región más competitiva en el sector turismo en el país, a pesar de que posee una escaza conectividad aérea, en comparación con otros departamentos (Pérez Pinzón, 2015).

En específico, se ha visto un aumento en el turismo del departamento, que ha pasado de recibir 600.000 visitantes anuales a 4.000.000 en el periodo 2002-2016. Ha llegado a obtener $\$ 120.000 .000$ COP en el año 2015, gracias al sector turismo. Esto demuestra que el sector tiene el potencial para crecer aún más y convertirse en la primera opción para los viajeros nacionales y extranjeros.

Según Pérez Pinzón (2015), se están desarrollando dos iniciativas estratégicas importantes para incentivar el sector en el mediano y largo plazo. La primera de ellas, que está en marcha, es la estrategia del Gobierno nacional de los Corredores Turísticos, de la que Santander hace parte, y a la fecha de la presente investigación se encuentra en la fase II (ver Figura 1). Esta fase hace referencia a los departamentos que tienen gran potencial para atraer demanda nacional pero que aún no atraen tantos turistas internacionales. 


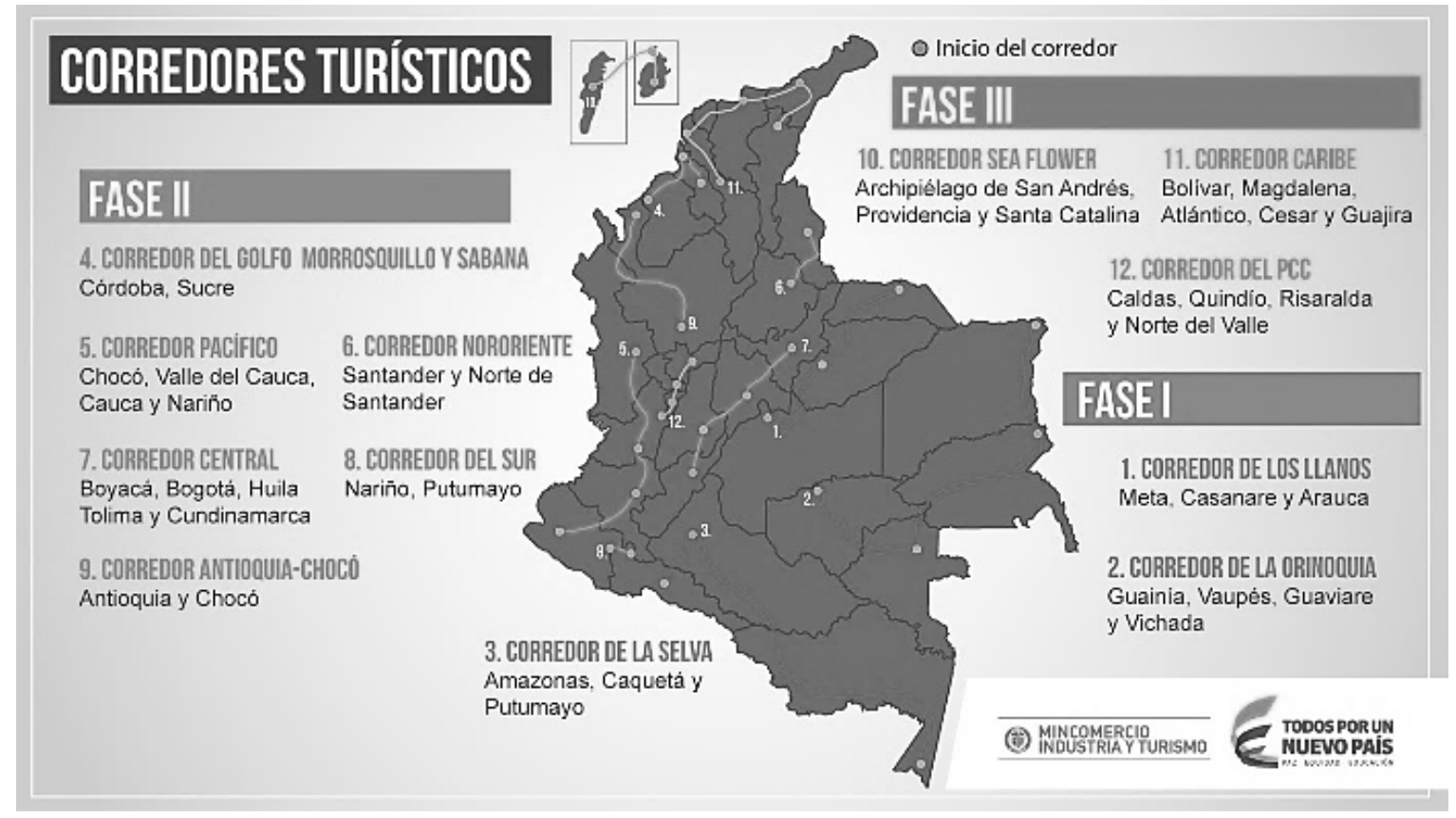

Figura 1. Corredores turísticos de Colombia. Fuente: Comisión regional de competitividad regional de Santander (2016).

Así mismo, en Santander existe una división de ocho corredores turísticos. La provincia García Rovira se encuentra en el Circuito del Paisaje del departamento, según el Plan de Desarrollo de los años 2004-2007, que corresponde a una de las regiones más competitivas para enfocarse en el turismo natural o ecoturismo (ver Figura 2).

La segunda estrategia se trata de la Gran Alianza por el Turismo de Santander 2030, con la cual se busca identificar y potencializar el desarrollo de este sector en el departamento. Se basa en cinco áreas importantes: educación, formación e investigación; productos y servicios; infraestructura; promoción y mercadeo; y fortalecimiento institucional. Esta estrategia se realizó por la unión de distintas instituciones públicas, privadas y académicas en pro del beneficio del sector.

Todas las cifras presentadas anteriormente se basan en datos generales del departamento de Santander, en comparación con los demás departamentos de Colombia. A la fecha de la presente investigación, no se hallaron datos concretos ni específicos sobre la competitividad del turismo en la provincia García Rovira.

\section{Método}

En el presente estudio se realiza una investigación exploratoria descriptiva con un enfoque cualitativo, dado que se realiza trabajo de campo para recolectar información acerca del turismo en los principales municipios de la provincia García Rovira, con el objetivo de reconocer su potencial turístico. Además, este estudio es de tipo transversal, porque se analizan datos de una muestra poblacional en un solo momento temporal.

El grupo poblacional en el que se enfoca el trabajo son los establecimientos de alojamiento. Adicionalmente, se consultó a expertos en turismo de la región, y se recurrió a búsqueda de material recopilado por otros estudios con anterioridad, de modo que se facilitara la comprensión de la estructura del sector turismo de la provincia. La selección de los establecimientos a encuestar se basa en una metodología de tipo no probabilística o dirigida, dado que se elige un subgrupo de establecimientos de alojamiento con las características requeridas para la investigación (Hernández Sampieri, Fernández Collado \& Baptista Lucio, 2010).

Se realizó un trabajo de campo en los municipios escogidos (Málaga, Capitanejo, Concepción y San Andrés), y así se determina el número de establecimientos a los que se les aplicó la encuesta. Basándose en la muestra no probabilística escogida para la recolección física de fuentes primarias, se realizan diez (10) entrevistas exhaustivas en los cuatro municipios, para conocer el estado actual del sector turismo y elaborar un diagnóstico. De igual forma, se realizan dos entrevistas con expertos de la provincia de Málaga y de la Asociación Colombiana de Aventura y Montaña.

Al momento del estudio, se identifican las condiciones 
para el sector turismo en los municipios de Málaga, Capitanejo, Concepción y San Andrés. Lo anterior en términos de hoteles disponibles, tipos de servicios adicionales, apoyo económico recibido y país de origen de los visitantes.

Para la realización de esta investigación, se implementa un diseño de teoría fundamentada. Hernández Sampieri, Fernández Collado y Baptista Lucio (2010) afirman que esta teoría se utiliza cuando "el estudio se basa en datos empíricos y se aplica en áreas específicas". Asimismo, la teoría fundamentada va más allá de los saberes previos y los conceptos preconcebidos, ya que busca nuevas maneras de concebir los procesos sociales que tienen lugar en ambientes naturales.

A partir de la información recolectada de las entrevistas, así como de la consulta a expertos, se realiza un diagnóstico del sector a través de la construcción de una matriz DOFA. Esto para determinar los niveles de competitividad del turismo en los municipios de Málaga, Capitanejo, Concepción y San Andrés, de la provincia García Rovira.

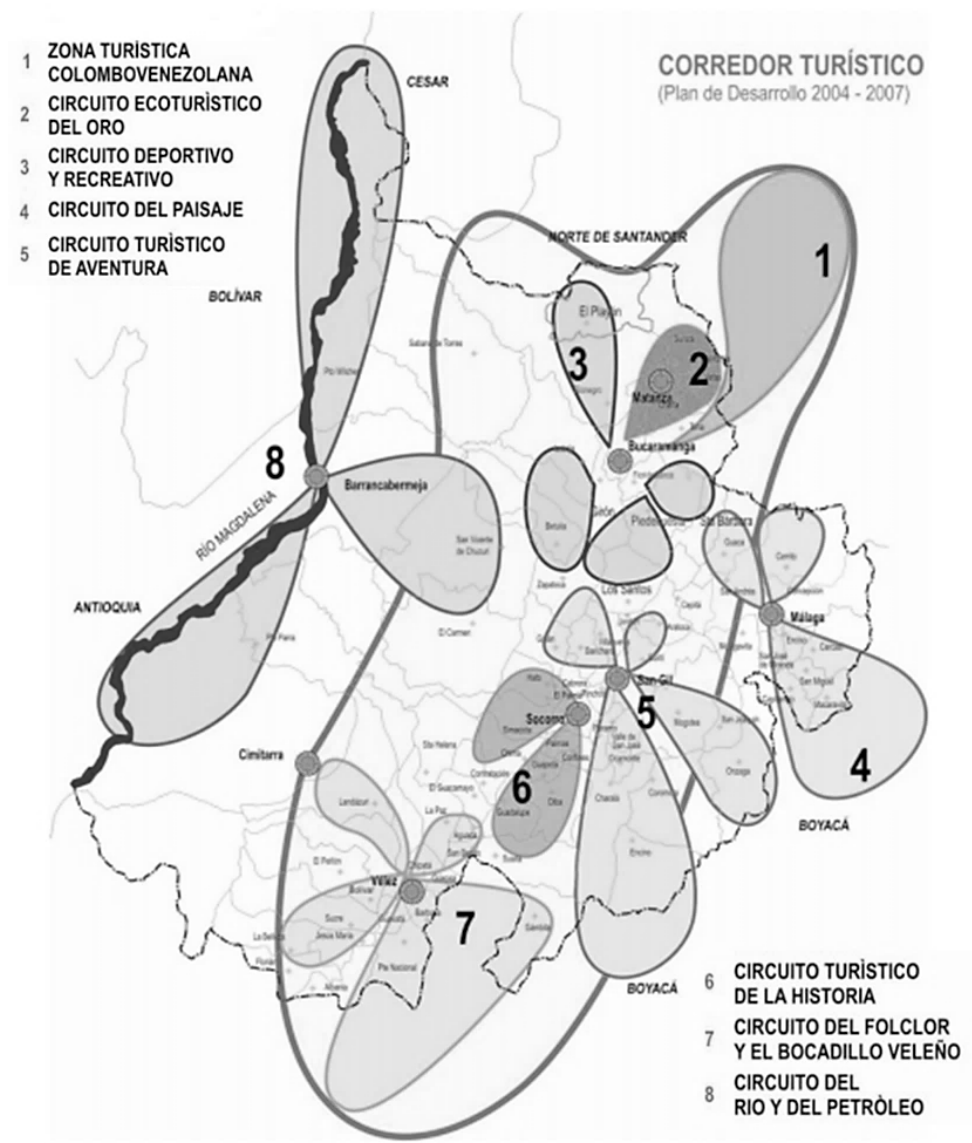

Figura 2. Corredor turístico del departamento de Santander. Fuente: Fondo de Promoción Turística de Colombia (2012).

\section{Resultados}

De las entrevistas realizadas en los cuatro municipios, específicamente a hoteles y centros de recreación que sirven como destino para el turista, así como de la consulta a expertos de la región, se destaca que el municipio de Málaga, capital de la provincia García Rovira, es el municipio con mayor número de sitios de hospedaje. En la Tabla 1 se presentan los municipios en estudio y el total de lugares de alojamiento registrados.
Entre los servicios adicionales prestados, solo el 29,6 $\%$ de estos establecimientos cuentan con restaurante, y el 11,1\% tienen áreas de recreación, como piscinas y balnearios. Según los dueños de hoteles y prestadores de servicios turísticos, lo anterior puede ocurrir por la falta de incentivos dados al sector por parte del Gobierno, ya que en la región solo el 11,1\% afirma recibir algún tipo de apoyo económico para mejorar sus servicios. 
Por otra parte, referente al lugar de origen de los visitantes, el $92,6 \%$ de los hospedados en estos municipios son de nacionalidad colombiana, mientras que el $7,4 \%$ son visitantes extranjeros, provenientes principalmente de países como Estados Unidos y España, con un $23,1 \%$ y $13,5 \%$, respectivamente. Con los resultados de estas encuestas, se evidencia que el sector turismo en esta región posee bajos niveles de competitividad, $y$, por ende, se procede a realizar un diagnóstico más detallado de este sector basado en la matriz DOFA.

En la Figura 3 se presenta la matriz DOFA. Allí se evidencia la relación entre las debilidades, oportunidades, fortalezas y amenazas, junto con el potencial turístico identificado en los municipios de
Málaga, Capitanejo, Concepción y San Andrés, de la provincia de García Rovira, del departamento de Santander.

\section{Tabla 1}

Sitios de alojamiento disponibles en los municipios de estudio

\begin{tabular}{cc}
\hline Municipio & Lugares de alojamiento \\
\hline Málaga & 12 \\
Capitanejo & 6 \\
Concepción & 2 \\
San Andrés & 7 \\
\hline
\end{tabular}

Fuente: Autores.

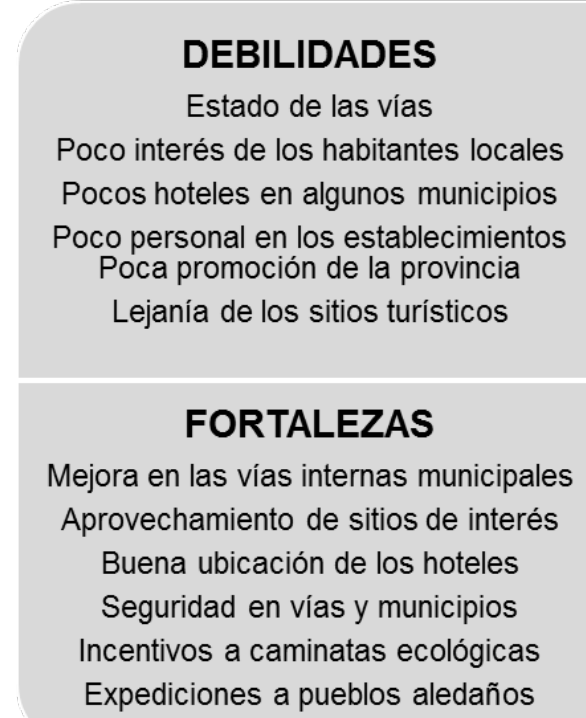

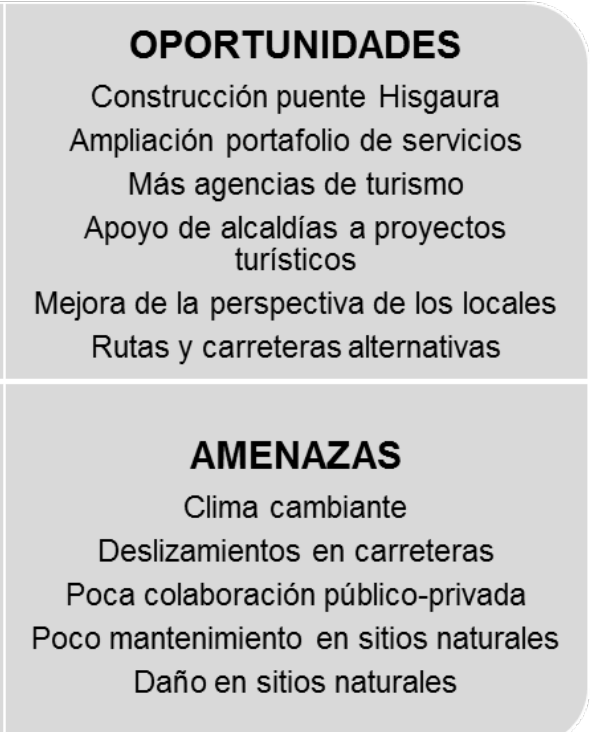

Figura 3. Matriz DOFA. Fuente: Autores.

\section{Relación debilidades-oportunidades}

Si bien el estado de las vías que comunican los municipios con la capital del departamento es bastante deficiente, existen rutas alternativas para atravesar la provincia que se encuentran completamente pavimentadas y en buenas condiciones. Tal es el caso de la vía Pamplona, para llegar desde Bucaramanga hasta Málaga.

Por otra parte, los habitantes de los municipios consideran que la provincia no es tan turística como otras. Sin embargo, esto puede mejorar con proyectos como el Chicamocha Run o el Balneario en Capitanejo, que necesitan mayor promoción de los medios de comunicación para convertirse en incentivos del turismo en la región.
Otras de las debilidades del sector son los pocos establecimientos de hospedaje y la ausencia de personal que atienda a los visitantes. Sin embargo, se esperaría que, al generarse un aumento paulatino de turistas en la provincia, se producirá más empleo en los establecimientos. De hecho, la provincia está siendo foco de inversiones en el área hotelera. Caso particular, el Balneario en Capitanejo, que ha empezado a contratar personas para atender a todos los clientes y finalizó recientemente la construcción de cabañas y zonas para camping.

\section{Relación debilidades-amenazas}

Una combinación bastante negativa son los deslizamientos y cambios climáticos que se presentan en la provincia con 
el estado de las carreteras. Estos factores se complementan para afectar el desarrollo del turismo en la región, lo que genera un aumento considerable en el tiempo de tránsito entre los municipios.

Sumado a esto, la ausencia de apoyo de los entes gubernamentales para la mejora del turismo ha generado desinterés de los habitantes de la región por sus atractivos turísticos. Esto se debe a la poca relación público-privada y del sector privado con el tema. Lugares naturales, como Aguas Calientes o la cascada en San Andrés, se encuentran en mal estado, pero los pobladores locales no están interesados en lo que le pueda ocurrir a la cascada, dado que no tienen los recursos ni las ayudas necesarias para tomar acciones que involucren la recuperación de estos sitios.

\section{Relación fortalezas-oportunidades}

Existen acciones relevantes que se están realizando en tema de infraestructura, como el arreglo interno de las carreteras de los municipios y la creación de macroproyectos de construcción, como el Puente Hisgaura. Esto se traduce en un avance en cuanto a la infraestructura de la región. Además, se han aumentado las visitas a algunos sitios turísticos de la provincia, para realizar actividades como la práctica de deportes acuáticos en la Laguna de Ortices, o caminatas ecológicas en los municipios y pueblos aledaños.

\section{Relación fortalezas-amenazas}

El apoyo a las caminatas ecológicas por parte de entes gubernamentales, especialmente en Málaga, capital de la provincia, junto con las expediciones naturales que atraviesan los pueblos aledaños del municipio (Enciso, Cerrito, Carcasí, entre otros), genera un incentivo indirecto a los demás municipios de la región. Además, hace ver al sector público que el turismo puede ser una alternativa más para el ingreso económico, y que la economía no solo se basa en el sector agrario.

Potencial turístico de los municipios de Málaga, Capitanejo, Concepción y San Andrés, de la provincia García Rovira

Los municipios de Málaga, Capitanejo, Concepción y San Andrés no son reconocidos por ofrecer variedad de sitios turísticos. Esto porque, desde hace varios años, esta actividad no ha recibido ningún incentivo para mejorar e invertir en las riquezas naturales con las que cuenta cada uno de los municipios mencionados.
Málaga, aun cuando es la capital de la provincia García Rovira, es un destino de paso para el viajero. La mayoría de las personas que visitan este municipio solo llegan a hospedarse, porque sus destinos son los municipios aledaños. Málaga posee un gran potencial hotelero, ya que cuenta con variedad de hoteles de buena calidad y a diferentes precios. Al ser la capital de la provincia, posee gran cantidad de establecimientos, como tiendas de ropa, calzado, restaurantes, cafeterías, supermercados y bares, entre otros.

Un atractivo turístico relevante que se destaca en este municipio es la realización del Chicamocha Run, que consiste en una carrera realizada anualmente por amantes del ecoturismo. La carretera recorre distancias que van desde los 9,5 km hasta los $183 \mathrm{~km}$. La carrera inicia en Málaga, recorre prácticamente toda la provincia García Rovira y atraviesa el cañón del Chicamocha, páramos y bosques. Se ha convertido poco a poco en uno de los principales atractivos para los amantes del deporte en los ámbitos nacional e internacional. Otro atractivo son los tours realizados para el avistamiento de cóndores en su hábitat natural, realizados por una agencia de viajes malagueña llamada "El Andinista", y que, además, incluye visitas a una comunidad indígena que habita cerca del municipio.

Por su parte, Capitanejo es el municipio más visitado por turistas de la provincia, ya que cuenta con diversidad de atractivos turísticos, como sus deportes extremos. Entre ellos, canotaje, parapente, barranquismo y puenting. Para aquellas personas amantes del ecoturismo, el municipio también tiene senderos ecológicos, miradores naturales, cascadas como el Salto de la Chorrera y el majestuoso cañón de Chicamocha. Este municipio también posee balnearios en lugares conocidos como las Cabañas Ceylan (ver Figura 4) y el centro recreacional y ecoturístico Palmeras y Brisas del Chicamocha.

Por su parte, Concepción, a pesar de ser un municipio pequeño, cuenta con un alto potencial turístico, al poseer interesantes sitios de aguas termales conocidas como "Aguas Calientes". Se trata de pozos naturales de aguas minerales que se originan en un volcán subterráneo, con temperaturas que van desde los $5{ }^{\circ} \mathrm{C}$ hasta los $42{ }^{\circ} \mathrm{C}$ (ver Figura 5). Debido a sus minerales, poseen grandes beneficios para la salud de los visitantes, que en su mayoría son personas con problemas de salud relacionados con reumatismo, dolores en las articulaciones, problemas en el sistema nervioso, enfermedades respiratorias, mala circulación sanguínea, entre otros. Adicionalmente, el barro 
contenido en los pozos sirve como tratamiento de belleza para el rejuvenecimiento de la piel.

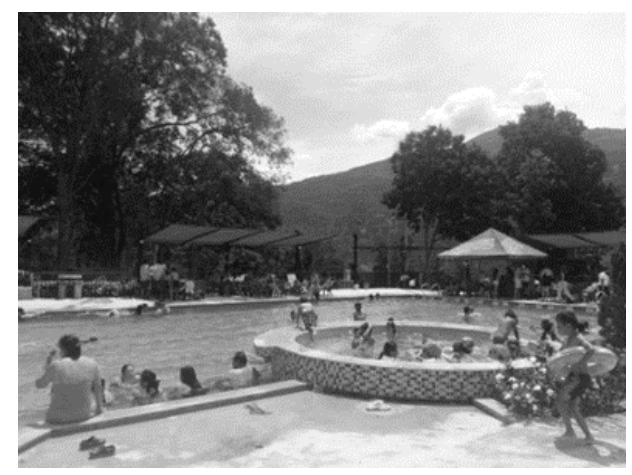

Figura 4. Balneario de Capitanejo. Fuente: Autores.

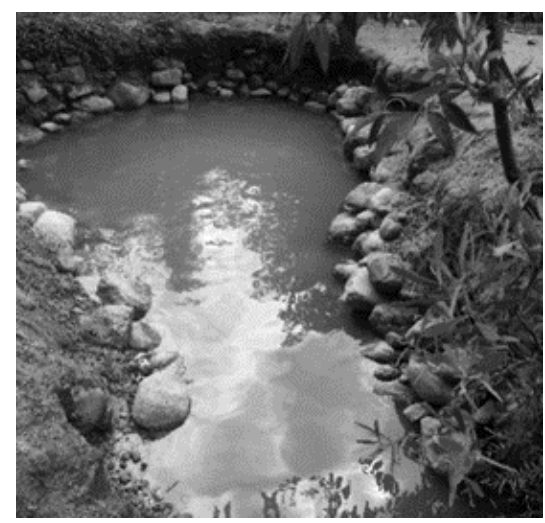

Figura 5. Pozo en Aguas Calientes. Fuente: Autores.

Otro atractivo turístico del municipio es el Sendero de la piedra de El Salado, un lugar apto para caminatas ecológicas. Se encuentra formado por rocas que adoptan distintos colores, gracias a la alta concentración de sodio encontrado en la región. Da cabida a la cascada de El Salado, una caída de agua con más de 20 metros.

Otro municipio que cuenta con un gran potencial turístico, especialmente en el ecoturismo, es San Andrés, ya que posee la tercera laguna más importante de Latinoamérica: la Laguna de Ortices, tal como se puede observar en la Figura 6. Esta laguna es el principal centro de atracción, con aproximadamente 25 metros de profundidad, una temperatura templada y hermosos paisajes. En ella se pueden realizar actividades como paseo en lancha de pedal, canoa y natación. Adicionalmente, en el municipio se pueden encontrar atractivos turísticos como observar el proceso de fabricación de panela tipo exportación, de forma artesanal, en los trapiches que se encuentran en el corregimiento.

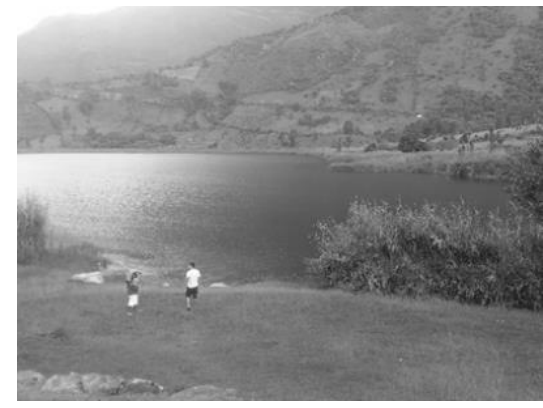

Figura 6. Laguna de Ortices. Fuente: Autores.

\section{Conclusiones}

En el desarrollo de la investigación se hizo evidente que son pocas las fuentes y bases de datos actualizadas con información básica de los municipios. A pesar de que cada municipio cuenta con una página web oficial dirigida por la alcaldía municipal que contiene información propia, los datos que se encuentran están desactualizados. Esto, a su vez, deja ver la importancia de usar tecnologías de la información y la comunicación que permitan, de una manera más amplia y actualizada, el fácil y rápido acceso a la información sobre el potencial turístico y los diferentes servicios que ofrece cada municipio.

Los municipios de Málaga, Capitanejo, Concepción y San Andrés cuentan con un potencial turístico alto, pero se debe invertir en la mejora de las vías de acceso y en su señalización, para obtener una mayor seguridad en las carreteras. De esta forma, la provincia García Rovira podría ser en un futuro un destino más deseado por los turistas.

Adicionalmente, se resalta la importancia de que las alcaldías y demás entes gubernamentales contribuyan con recursos y acciones que impulsen el desarrollo del sector turismo en cada uno de los municipios. Para ello, son necesarias la elaboración y ejecución de más proyectos y planes de desarrollo; asimismo, es indispensable que los recursos destinados para el turismo se inviertan en las áreas clave del sector, entre ellas la de infraestructura.

Cabe resaltar la puesta en marcha de proyectos como el Chicamocha Run, que está recibiendo una respuesta favorable por parte de los habitantes y gobernantes, quienes incluso están haciendo convenios con la Asociación Colombiana de Aventura y Montaña, para seguir incentivando la carrera. Este es un paso más en la dirección correcta, así como una muestra evidente de que, aunque tomó un poco más de tiempo que 
en otras provincias, García Rovira finalmente está despertando ante la posibilidad de ser más turística; ya no se enfoca netamente en sus actividades agrícolas.

Además, la diversidad de recursos naturales que ofrecen los cuatro municipios potencia el turismo. Estos recursos se traducen en grandes oportunidades que se pueden convertir en fuentes de ingresos que contribuyan con el desarrollo de los municipios y de la provincia. De esta forma, la región se hace más competitiva frente a otras del departamento de Santander, y se puede convertir en un exponente del turismo natural, tanto en el ámbito departamental como en el nacional y el internacional.

\section{Recomendaciones}

La información que suministran las páginas de cada alcaldía no es muy específica y amplia. Esto hace que no se conozcan con profundidad las oportunidades y sitios de interés que posee cada municipio. Por tanto, para impulsar el desarrollo del turismo en estas regiones, se hace un llamado a las alcaldías para que tomen acciones que tiendan al mejoramiento de la imagen de cada municipio, así como a la difusión de los atractivos turísticos que ofrecen. Esto se logra si se mantiene la información actualizada en las páginas de cada entidad, de forma que a las personas les resulte más fácil el acceso a información actualizada.

La medición de la competitividad turística es compleja, debido principalmente a su carácter subjetivo y a la complejidad de definir el entorno de los lugares objeto de estudio. Para el desarrollo de futuras investigaciones, se recomienda analizar la competitividad turística en otros municipios del departamento -o de uno diferente-, incluyendo otras variables, como capital humano y turístico, factores culturales y precio/calidad.

\section{Agradecimientos}

Al Sr. Freddy Rincón, policía de turismo de Málaga, quien brindó información sobre los municipios de la región y dio una guía sobre los sitios de interés.

Al Sr. Elkin Yesid Quintana, fundador de la Asociación Colombiana de Aventura y Montaña (ACAM) y organizador del Chicamocha Run, quien dio su opinión sobre el potencial turístico y proporcionó imágenes sobre la ruta realizada en la región.

Al Sr. Cristian Fabián Medina Ardila, quien sirvió como guía y transportista durante todo el recorrido por los municipios.

\section{Referencias}

Botero, C., \& Zielinski, S. (2010). Evaluación del potencial para el desarrollo de turismo sostenible en el corregimiento de Taganga, distrito de Santa Marta (Colombia). Turismo y Sociedad, 11, 10-34.

Cardoso Jiménez, C. (2006). Turismo Sostenible: una revisión conceptual aplicada. El Periplo Sustentable, 11.

Castro, M., \& Robles, L. (2018). Laboratorios de logística internacional como modelo de competitividad aplicado en las universidades de Colombia. $I+D$ Revista de Investigaciones, 13(1), 88-97. https:// doi.org/10.33304/revinv.v13n1-2019009

Comisión regional de competitividad regional de Santander. (2016). El Turismo en Santander, sus Números y su Proyección. Bucaramanga.

Faraldo, J. M., \& Rodríguez-López, C. (2013). Introducción a la historia del turismo (1st ed.). Madrid, España: Alianza Editorial.

Fayos-Solá, E. (1994). Competitividad y calidad en la nueva era del turismo. Estudios Turísticos, 123, 5-10.

Financial Times. (2016). South American States of the future 2016 - 2017. São Paulo, Brasil.

Fondo de promoción turística de Colombia. (2012). Unión temporal turismo cundisán. Bogotá. Retrieved from http://www.mincit.gov.co/ CMSPages/GetFile.aspx?guid=ae18c3ad-0b954e45-ae6e-a3328d3f038f

García, M. O., Ramírez, I. L., \& González, C. V. (2018). Tendencias del turismo hasta 2030. Anudar Red. Temas Pendientes y Nuevas Oportunidades de Cooperación en Turismo, 107-127. https://doi. org/10.17345/9788484244905

González, R. C., \& Mendieta, M. D. (2009). Reflexiones sobre la conceptualización de la competitividad de destinos turísticos. Cuadernos de Turismo, 23, $111-128$

Hernández Sampieri, R., Fernández Collado, C., \& Baptista Lucio, M. del P. (2010). Metodología de la investigación. (McGraw-Hill / Interamericana Editores, Ed.) (5.a ed.).

Hong, W. C. (2009). Global competitiveness measurement for the tourism sector. Current Issues in Tourism, 12(2), 105-132. https://doi. org/10.1080/13683500802596359

Manrique, O. (2013). Verdades en las iniciativas de clúster en el departamento de Santander. $1+D$ Revista de Investigaciones, 2(2), 29-40. https://doi. org/10.33304/revinv.v02n2-2013003

Organización Mundial de Comercio. (2016). Examen Estadístico del Comercio Mundial. Ginebra, Suiza.

Organización Mundial del Turismo. (2015). Historia Organización Mundial del Turismo. 
Pérez Pinzón, L. (2015). Characterization of Sources of Financing for Tourism Sector in Santander (Colombia). Turismo y Sociedad, 17.

Porras Celis, M., \& Ruiz Pico, G. I. (2005). Factibilidad para la creación de una empresa de turismo ecológico en el municipio de suaita santander. Universidad Industrial de Santander.

Rice, E. A. B. (2018). El papel de la Ventaja Competitiva en el desarrollo económico de los países. Revista Análisis Económico, 28(69), 55-78.

World Economic Forum. (2018). Índice de Competitividad Global.

Zuñiga Collazos, A. (2015). Análisis de la investigación y desarrollo turístico en Colombia. Revista Espacios, 36(18). 